\title{
Early stages of motor skill learning and the specific relevance of the cortical motor system - a combined behavioural training and theta burst TMS study
}

\author{
Thomas Platz $^{\mathrm{a}, *}$, Sybille Roschka ${ }^{\mathrm{a}}$, Marianne I. Christel ${ }^{\mathrm{a}}$, Felix Duecker ${ }^{\mathrm{b}}$, John C. Rothwell ${ }^{\mathrm{c}}$ and \\ Alexander Sack ${ }^{\mathrm{b}}$ \\ ${ }^{a}$ BDH-Klinik Greifswald, Neurorehabilitation Centre and Spinal Cord Injury Unit, Neuroscience Department, \\ Ernst-Moritz-Arndt-Universität, Greifswald, Germany \\ ${ }^{\mathrm{b}}$ Department of Cognitive Neuroscience, Faculty of Psychology and Neuroscience, Maastricht University, \\ Maastricht, The Netherlands \\ ${ }^{\mathrm{c}}$ Sobell Department of Motor Neuroscience and Movement Disorders, Institute of Neurology, Queen Square, \\ London, UK
}

\begin{abstract}
Purpose: To examine whether motor performance and motor learning in healthy subjects can be segregated into a number of distinct motor abilities which are linked to intact processing in different motor-related brain regions (M1, S1, SMA, PMC) early during learning.

Methods: Seven young healthy subjects trained in eight motor arm tasks (Arm Ability Training, AAT) once a day for 5 days using their left non-dominant arm. Except for day 1 (baseline), training was performed before and after applying an inhibitory form of repetitive transcranial magnetic stimulation (cTBS, continuous theta burst) to either M1, S1, SMA, or PMC.

Results: A principal component analysis of the motor behaviour data suggested four independent motor abilities: aiming, speed, steadiness, and visuomotor tracking. AAT induced substantial motor learning across abilities. Within session effects of cTBS revealed that activity in primary somatosensory cortex (S1) was relevant for motor performance and learning across all tasks whereas M1 was specifically involved in rapid tapping movements, PMC in ballistic arm navigation in extra-personal space; performance on a non-trained motor tasks was not affected by cTBS.

Conclusions: Cortical sensory and motor areas including S1, M1, and PMC functionally contribute to early motor learning in a differential manner across motor abilities.
\end{abstract}

Keywords: Motor practice, learning, cortex, plasticity, transcranial magnetic stimulation

${ }^{*}$ Corresponding author: Prof. Dr. med. Thomas Platz, BDHKlinik Greifswald, An-Institut der Ernst-Moritz-Arndt-Universität, Karl-Liebknecht-Ring 26a, D-17491 Greifswald, Germany. E-mail: t.platz@bdh-klinik-greifswald.de.

\section{Introduction}

Motor plasticity involves a range of different processes that occur over time scales lasting from a few minutes to days or weeks. This involves strengthening existing synapses, growing new synapses, and then eliminating and consolidating the most efficient 
connections to drive successful performance of a task. These short and long term stages of learning lead to detectable changes in brain anatomy and physiology (Jäncke, 2009).

For example, it has recently been shown that $40 \mathrm{~h}$ of golf practice, performed as a leisure activity with highly individual training protocols is associated with increased grey matter volume in a task-relevant cortical network encompassing sensorimotor regions and areas belonging to the dorsal stream (Bezzola et al., 2011). Shorter term skill training can lead to changes in the pattern of task related activity in relevant brain networks. For example, Park et al. (2010) used functional MRI (fMRI) to chart changes in brain activation during short-term motor skill learning of a sequential finger movement in a range of motor areas including primary motor cortex (M1), primary somatosensory cortex (S1), premotor cortex (PMC), the supplementary motor area (SMA), the posterior parietal cortex and the cerebellum.

M1, PMC, SMA, and S1 all appear to be particularly important in the early stages of motor skill acquisition (Ashe et al., 2006; Grafton et al., 2002; Karni et al., 1995; Pascual-Leone et al., 1994; Vidoni et al., 2010). Thus, motor learning can be abolished if M1 is disrupted by repetitive transcranial magnetic stimulation (rTMS) applied directly after training (Muellbacher et al., 2002; Baraduc et al., 2004), while there is no effect if applied after several hours. Similarly, disruptive rTMS of $\mathrm{S} 1$ prior to practice of a continuous tracking task has also been shown to impair motor learning, an effect attributed to the development of an inaccurate internal model of the task (Vidoni et al., 2010).

The present experiments were designed to test whether different parts of this distributed network are preferentially involved in learning particular types of movement skill. To do this, we examined motor learning in the Arm Ability Training (AAT) task, a protocol that has been designed to train in parallel different sensorimotor abilities such as speed, aiming, dexterity, tracking and steadiness (Platz, 2004). It is a highly structured, standardised training and uses eight different training tasks that are trained each day in parallel: aiming, tapping, crossing circles, turning coins, labyrinth tracking, bolts and nuts, placing small objects, and placing large objects (Fig. 1).

It is clinically effective (Platz et al., 2001) and has superior clinical effectiveness when compared to individually tailored best conventional therapy as shown in a recent multicenter trial (Platz et al., 2009). Thus, parallel training of different motor tasks that are believed to train different sensorimotor abilities induces substantial motor re-learning after brain damage.

We suggest that the different sensorimotor abilities that are trained with AAT can be viewed as "classes" of motor skills, which in schema theory are represented as "generalised motor programs" (GMP) that store the invariant features that characterise the skill (Maas et al., 2008; Keetch et al., 2008). The GMP allows us to adjust its parameters to suit specific environmental demands (Schmidt, 1975) so that we can perform a task in variety of conditions whether previously experienced or completely novel. We hypothesise that different regions of the sensorimotor network are preferentially involved in forming the GMPs obtained through practice. The present experiments test this by using disruptive rTMS over different cortical areas during training sessions to test whether interference with specific parts of the network disrupts the acquisition of particular skills.

The current study was designed to test the hypotheses that i) parallel training of different motor tasks (AAT) induces motor learning and addresses distinct motor abilities, and ii) that these abilities map onto preferential involvement of different motor areas of the brain during early motor learning, i.e. before motor skills have been overlearnt after extended practise.

For this purpose, right-handed healthy AAT-naive subjects trained daily for 5 consecutive days on a standardised AAT task with their non-dominant left arm. First, we assessed in healthy subjects whether AAT induced motor skill learning, i.e. improvement of performance within each training session and more importantly, more robust learning across days. Furthermore we examined whether performance was coupled across motor tasks, suggesting the presence of a general motor factor (for arm motor control) or was different between tasks, indicating that separate motor abilities exist that at least to some extent could be trained independently. Finally we investigated whether an excitability reducing, inhibitory rTMS protocol (continuous theta burst stimulation with a total of 600 stimuli, cTBS-600) influenced motor skill acquisition within training sessions, and if so, whether the effect was different when applied over different motorrelated brain regions (M1, SMA, PMC, S1). The aim was to test if the effect of stimulation site was specific for the different trained motor tasks (and thus motor abilities) or was general in nature. 

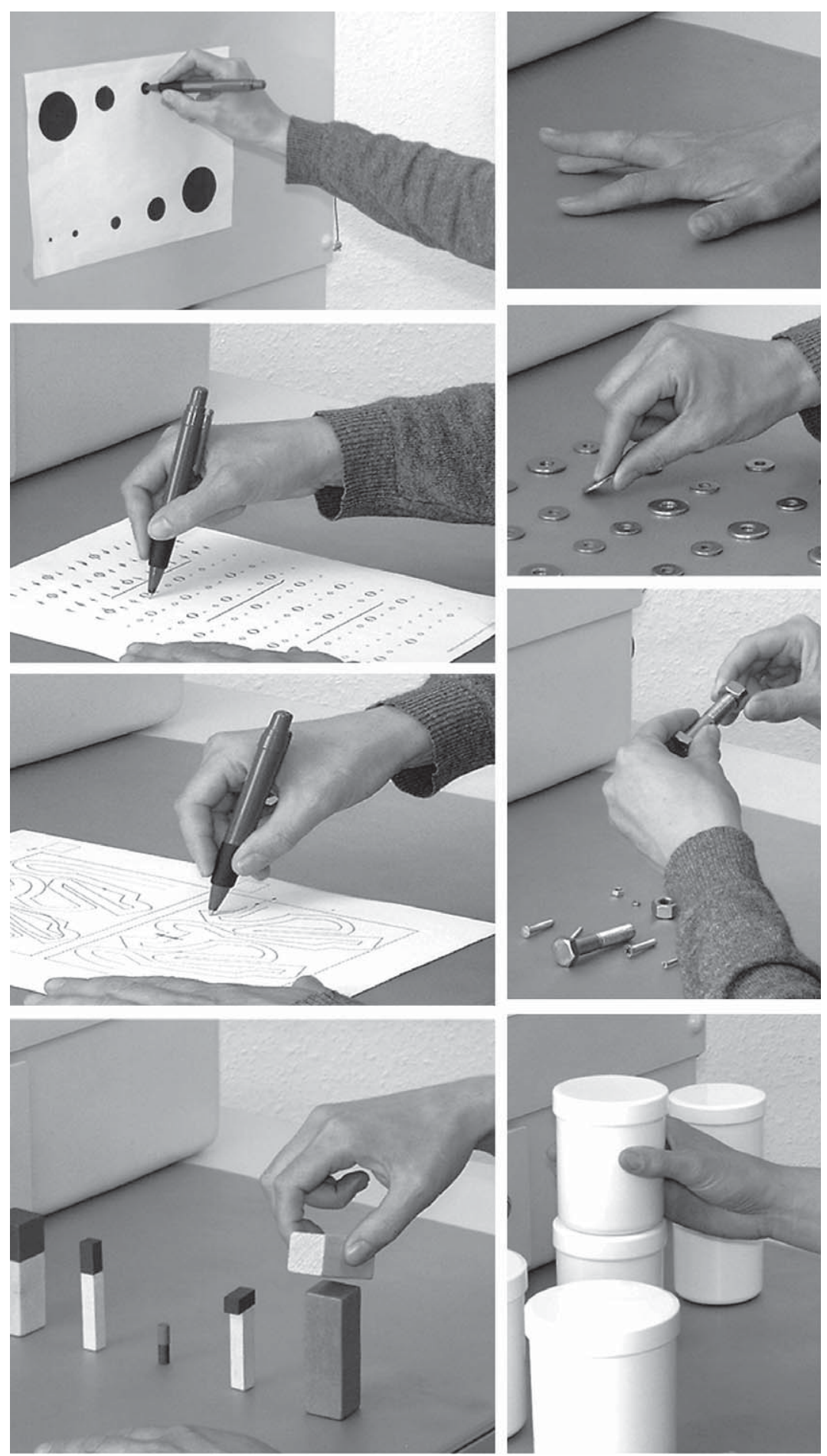

Fig. 1. Arm Ability Training tasks. The Arm Ability training consists of 8 different motor tasks that are thought to train different abilities such as speed, aiming, dexterity, tracking, steadiness and dexterity. The figure presents the training tasks that are practiced with a fixed sequence within each training session: the tasks are aiming, tapping, crossing circles, turning coins, labyrinth tracking, bolts and nuts, placing small objects, and placing large objects. 


\section{Materials and methods}

\subsection{Subjects}

Seven right-handed volunteers of (5 women, 2 men; mean age 23.6 \pm 3.2 years; Oldfield Handedness laterality ratio: $93 \pm 5$ ) participated and completed the study. All participants had normal or corrected-to-normal vision and had no history of neurological or psychiatric disorders. With regard to their past motor experience, all participants represent a non-selective sample of AAT-naive healthy volunteers. They received medical approval for participation and gave their written informed consent after being introduced to the procedure. The study was approved by the local Medical Ethical Commission of Maastricht University, where the experiment was conducted.

\subsection{Overall study design}

Participants were tested in six separate sessions. In the first session, we obtained anatomical brain measurements of all participants using magnetic resonance imaging (MRI). We performed a surface reconstruction to recover the spatial structure of the cortical sheet based on the white-grey-matter boundary. We then identified four motor-related brain regions, namely M1, S1, SMA, and PMC, on the basis of each individual brain gyrification (M1 was determined based on a combination of anatomical and motor electrophysiological data; for details see TMS procedure). In the second session, participants performed the AAT as a baseline measurement (see section on Motor tasks and training). In the following four sessions, participants performed the arm ability training before and after having received cTBS to one of the four above mentioned motor-related target sites using MRI-guided TMS neuronavigation. The order of stimulation site was counterbalanced across subjects. This study design and methodological approach enabled us to first define the target brain area based on the individual anatomical data and to subsequently neuronavigate the TMS coil to the anatomically defined stimulation sites in each participant. The MRI-guided TMS neuronavigation was monitored online, allowing for a precise determination of the actual stimulation site during cTBS.

\subsection{Motor tasks and training}

The AAT consists of 8 different motor tasks that are thought to train different abilities such as speed, aiming, dexterity, tracking and steadiness: the training tasks with a fixed sequence within each training session are aiming, tapping, crossing circles, turning coins, labyrinth tracking, bolts and nuts, placing small objects, and placing large objects (Fig. 1) (Platz, 2004).

Work load had been standardised for all participants in advance: every day the same amount of task repetitions had to be trained. The specified number of repetitions for each task per day had been divided in four equal "blocks" (fixed number of repetitions lasting approximately 1 minute at the beginning). During training, participants were continuously encouraged to try to fulfil their workload in even shorter time, but without compromise of the individual tasks' accuracy demands. The accuracy demands of the tasks were kept constant; thus, any improvement in skilfulness would translate into a reduced time needed to complete the tasks repetitions. Any progress was shown to participants for each type of task during training sessions using diagrams on a PC screen (Arm Ability software; knowledge of result, i.e. time needed for blocks of task repetitions). Commercially available training material and documentation software was used (for reference see http://www.iotraining.eu/material.html). Participants repeated a total of 4 blocks of repetitions for each training task per day in two consecutive training slots with two combined blocks for each task for all tasks (1st half of training session) followed by another two combined blocks for each task for all tasks (2nd half of training session) (Fig. 2). The same standardised training was applied for 5 weekdays, each training lasting appr. 1 hour.

In addition, the Nine-Hole-Peg-Test was administered as a standard motor test for both hands after the AAT tasks, both before and after cTBS (for cTBS details see below).

\subsection{TMS procedure}

cTBS was used to reduced the excitability of either M1, S1, PMC or SMA temporarily during training sessions on day 2 to 5 . These targets for cTBS had been determined neuroanatomically on an individual basis using anatomical MRI scans, cTBS to these targets was supported by neuronavigation in each individual participant. 

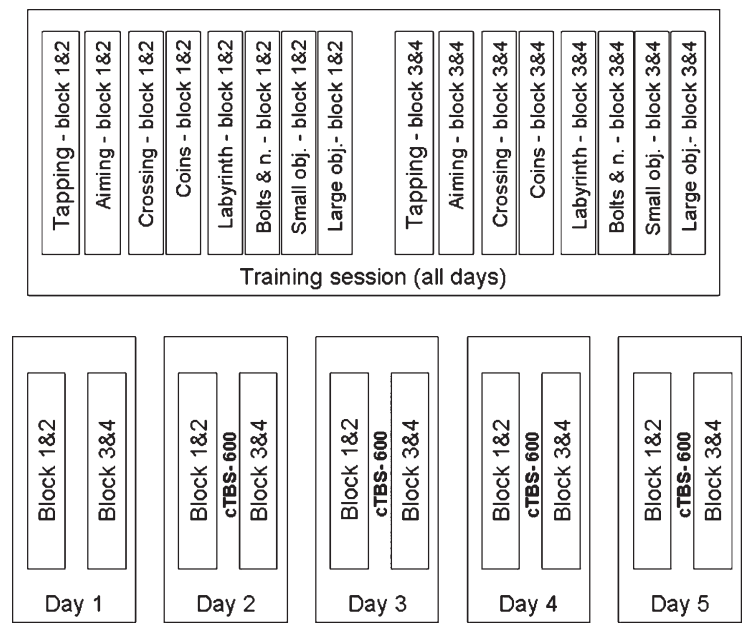

Fig. 2. Standardised sequence of motor training. The participants repeated a total of 4 blocks of repetitions for each training task per day in two consecutive training slots with two combined blocks for each task for all tasks (1st half of training session: blocks 1 \& 2) followed by another two combined blocks for each task for all tasks (2nd half of training session: blocks $3 \& 4$ ). This structure was repeated for five consecutive days. On days 2 to 5, cTBS was applied between the 1 st and 2 nd half of each training session.

Each participant's anatomical MRI was imported into BrainVoyager ${ }^{\mathrm{TM}}$ TMS neuronavigation software (TMS Neuronavigator edition of Brain Voyager QX 2.1 by Brain Innovation B.V., Maastricht, NL) and used for stereotaxic co-registration of the participant's brain with the TMS coil. This enabled online control and retest-reliability of coil positioning during each session and across days. Subjects were seated in a reclining chair and instructed to remain relaxed throughout the application of rTMS. Surface electromyography (EMG) from participants' abductor pollicis brevis muscles (APB) was monitored using the motor evoked potential unit of (Dantec Keypoint ${ }^{\circledR}$ by Alpine Biomed ApS, Skovlunde, DK).

Application of TMS was performed with a $70 \mathrm{~mm}$ figure-8 air-cooled coil (Magstim Rapid stimulator, Magstim Company, Ltd., Wales, UK). The TMS coil was oriented tangentially to the scalp with the handle pointing back and away from midline at $45^{\circ}$ during stimulation of both M1 (for thresholding and cTBS), S1 (for cTBS), and PMC (for cTBS) while it was oriented tangentially to the scalp with the handle pointing downward and away from midline at $90^{\circ}$ during stimulation of the SMA (for cTBS).
Motor evoked potentials (MEPs) were used to determine the coil position over M1 ("hot spot") that evoked the best response in the left (and right) APB. The location of this spot was marked using BrainVoyager ${ }^{\mathrm{TM}}$ to minimize variability across subsequent trials and days. The other stimulation sites of the right brain were defined in the individual MR data: PMC, in the middle of the gyrus immediately frontal to M1 on a line perpendicular to the central gyrus through the hot spot; S1, in the middle of the gyrus immediately posterior to $\mathrm{M} 1$ on a line perpendicular to the central gyrus through the hot spot; SMA, $3 \mathrm{~cm}$ anterior from the leg motor area (mid of precentral gyrus $1 \mathrm{~cm}$ lateral to midsagittal line) and $1 \mathrm{~cm}$ lateral to the midsagittal line. The locations of M1, S1, PMC and SMA were maintained both within and across sessions by use of the Brain Voyager ${ }^{\mathrm{TM}}$ TMS neuronavigation (Fig. 3).

Each cTBS session consisted of a continuous $40 \mathrm{sec}-$ ond train of TBS with 600 stimuli (cTBS-600). cTBS protocols apply short bursts (3 stimuli) of $50 \mathrm{~Hz}$ rTMS which are repeated at a rate in the theta range $(5 \mathrm{~Hz})$. Because the bursts are given at high frequency, the intensity of stimulation should be quite low, and certainly below resting motor threshold for safety reasons (Rossi et al., 2009). In this experiment, an intensity that equals $80 \%$ of the active motor threshold (AMT) was applied; the AMT being the intensity that evokes an MEP of $\geq 200 \mu \mathrm{V}$ in $\geq 5$ out of ten trials while the subjects perform an isometric contraction at a level of $20 \%$ of her/his maximum voluntary contraction (Huang et al., 2005). Applied continuously (cTBS), the net effect of TBS is inhibitory, cTBS-600 can temporarily suppress local cortical excitability for about 60 minutes (Huang et al., 2005).

Both MEP amplitudes and cortical latency of MEPs for the left (and right) APB muscle had been determined at baseline (day 1) and before and after each cTBS intervention (days 2 to 5).

\subsection{Data analysis}

A principal component analysis (PCA) was used to assess the data structure of the behavioural AAT data. For this purpose, the behavioural data for all training tasks and each block of performance (5 days with 4 blocks of training for each training task) had been analysed. Here it was of interest to assess empirically whether independent components could be detected in the data set of these 152 variables (tasks $(i=8) \times$ blocks $(i=4) \times$ days $(i=5)$; except for 

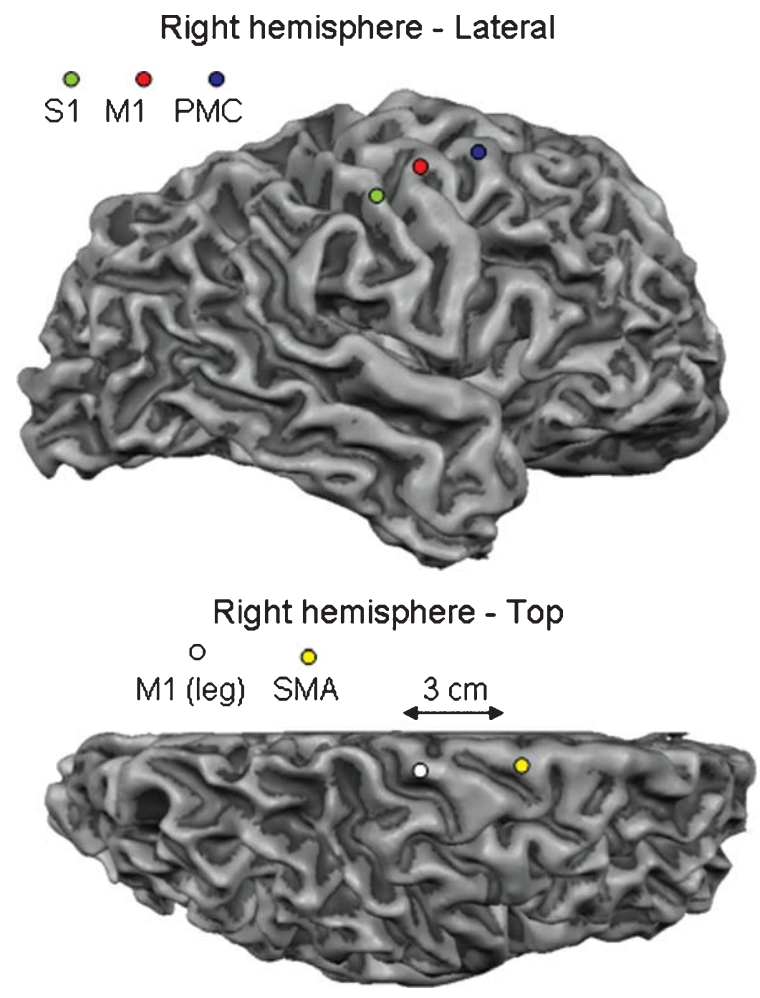

Fig. 3. TMS neuronavigation. Motor evoked potentials (MEPs) were used to determine the coil position over M1 ("hot spot") that evoked the best response in the left (and right) APB. The location of this spot was marked in the individual MR-based brain model using BrainVoyager TM to minimize variability across subsequent trials and days (compare "M1" in figure). The other stimulation sites of the right brain were defined in the individual MR data: PMC, in the middle of the gyrus immediately frontal to M1 on a line perpendicular to the central gyrus through the hot spot; S1, in the middle of the gyrus immediately posterior to M1 on a line perpendicular to the central gyrus through the hot spot; SMA, $3 \mathrm{~cm}$ anterior from the leg motor area (mid of precentral gyrus $1 \mathrm{~cm}$ lateral to midsagittal line) and $1 \mathrm{~cm}$ lateral to the midsagittal line. The locations of M1, S1, PMC and SMA were maintained both within and across sessions by use of the BrainVoyager ${ }^{\mathrm{TM}}$ TMS neuronavigation.

block 1 of day 1 for each task that was used for standardisation [=1]). The PCA could e.g. show a single general motor component or alternatively, could group variables according to the assumed five independent abilities such as speed, aiming, dexterity, tracking and steadiness. Since we used PCA to extract uncorrelated components in the data set a varimax rotation was used. The number of uncorrelated principal components to be retained and rotated was pre-specified with 5 since five independent abilities were theoretically assumed, components retained should have an eigenvalue of at least 1.0.
The behavioural outcome measures were the time needed for each block (blocks 1 to 4 ) for each training task (tasks 1 to 8) for each day of practice (days 1 to 5). These outcome measures (in seconds; appr. 60 seconds per block) had been standardised with baseline data from day 1 (i.e. block 1 for each task on day 1); thus, baseline data for each tasks were 1.0; any improvement in time needed to perform the training tasks would reduce the standardised block measure below 1.0. For the analysis of motor learning effects, these standardised scores for blocks of training tasks were used. Thereby, a simultaneous statistical analysis of any training effects within training sessions (across blocks), across days, and across training tasks was possible.

Since cTBS was applied on days 2 to 5 between the first and second half of the training session (i.e. between blocks $1 \& 2$ and $3 \& 4$ for each task), effects of cTBS were analysed based on these differences: For each day and task, difference scores between blocks $1 \& 2$ and $3 \& 4$ were calculated and standardised (with baseline data). Site of stimulation (i.e., M1, S1, PMC, SMA) had been counterbalanced across subjects. Thus, for testing the effects of stimulation per site, pre and post stimulation data (blocks $1 \& 2=$ pre stim, blocks $3 \& 4=$ post stim) and their differences were calculated for each site of stimulation and each training task.

General linear models within a repeated-measures ANOVA design were used to assess both the effect of (a.) training and (b.) cTBS on outcome measures: Repeated measures were either (a.) standardised time scores for blocks of training tasks or (b.) pre vs. post stim differences for the cTBS sites.

As stated above, effects of cTBS site were analysed as within session change scores from block $1 \& 2$ to block $3 \& 4$. Embedded in this repeated measures ANOVA design were specific contrasts between each stimulation site and the mean of all other stimulation sites (transformation matrix) and thus specific differences for a given stimulation site were determined: all other stimulation sites served as control condition for each stimulation site.

For the non-trained motor tasks, the Nine-Hole-PegTest, a repeated-measures ANOVA design was used to assess the effect of training across days ( 1 to 5), within sessions (after 1st and 2nd half of training session), and hand (left versus right) on the timed performance.

$F$ values presented for these models are partial $F$ values (based on type III sums of squares). Effect sizes "d" 


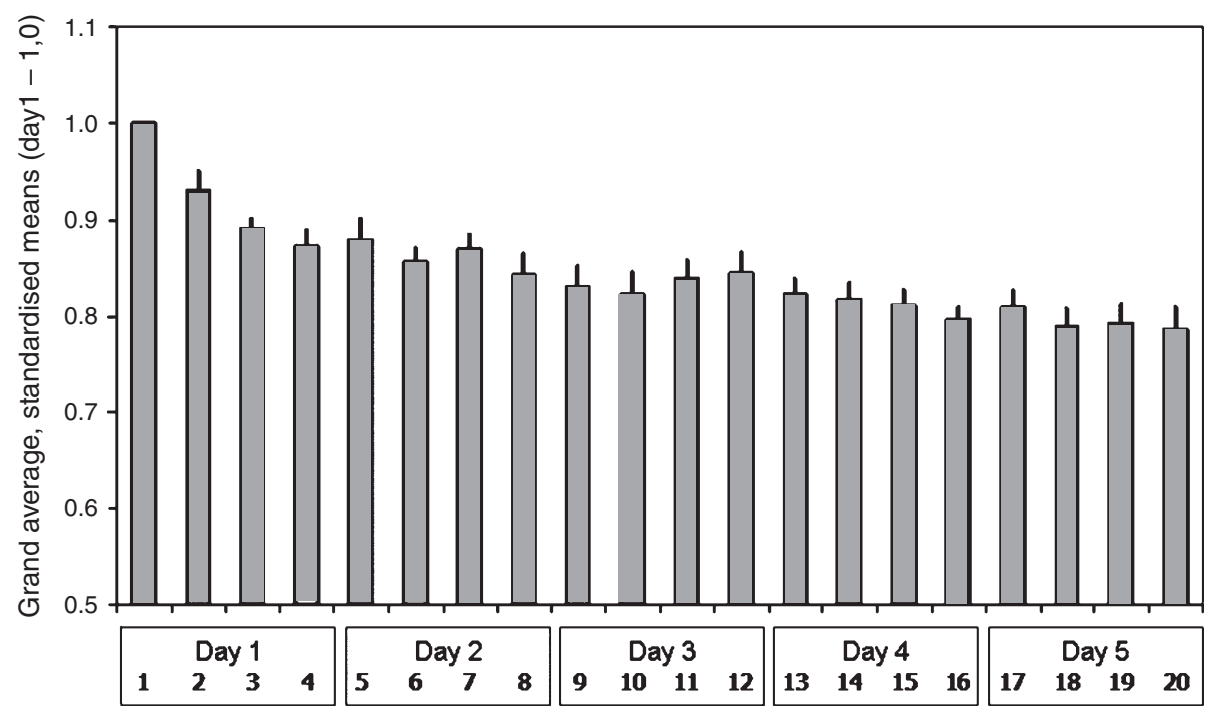

Fig. 4. Motor learning. The figure presents grand average data across all eight Arm Ability tasks; data is presented as mean and standard error of the mean (error bars) for "blocks" of task repetitions. During each training session, 4 blocks of task repetitions had been trained for each motor task. Data had been standardised for each task with its baseline data (block1) before averaging across tasks. The analysis of these changes corroborated that motor learning occurred, and more specifically, both within sessions (across blocks) and across sessions (over days).

had been calculated for statistically significant main effects of interest based on these F statistics; by convention, they had been considered small $\mathrm{d}=0.2$, medium $\mathrm{d}=0.5$, or large $\mathrm{d}=0.8$ (Cohen, 1988). In case assumptions of the repeated measures ANOVA were violated (according to sphericity testing), Huynh-Feldt epsilon adjusted $p$ values (labeled "H-F") were used. Alpha was set at 0.05 .

\section{Results}

\subsection{Principal component analysis}

The PCA of the AAT tasks revealed a meaningful 5-component solution with communalities for each factor ranging from 21.4 to 31.9 and a total communality estimate of 139.9 for the model. This suggests a fairly high degree of independence of groups of variables in the data set. Table 2 depicts the distribution of loadings matrix of the 8 Arm Ability training tasks on the five components as identified by PCA ( 4 blocks $\times 5$ days [except block 1 on day 1] = 19 variables per task). The data shows a high loading of component 1 on aiming, of component 2 on crossing circles, of component 3 on tapping and 'bolts and nuts', and of component 4 on the labyrinth task.

\subsection{Motor learning}

\subsubsection{Arm ability tasks}

The analysis of changes in task performance (based on all tasks, blocks, and days) indicated that motor learning occurred (Fig. 4) both within sessions (across blocks) $(\mathrm{F}(3,18)=19.43 ; p<0.0001)$ and across sessions (over days) $(\mathrm{F}(4,24)=31.16$; $p<0.0001 ; \mathrm{d}=2.28$ ) with effects over blocks being different for different days $(\mathrm{F}(12,72)=7.70 ; p<0.0001)$, i.e. becoming less as training progressed.

Interestingly the dynamics of motor skill learning was different across tasks as indicated by a significant task $\times$ block interaction $(\mathrm{F}(21,126)=4.06 ; p<0.0001)$ and a main effect of task $(\mathrm{F}(7,42)=4.49 ; p=0.0049)$ (even though all data had been standardised with performance of block 1 of day 1 for each task), suggesting a varying degree of skill level across tasks after baseline. Table 1 shows the different levels of performance at the end of training. Tapping showed the least improvement, crossing circles the largest dynamics in motor skill learning over 5 days.

\subsubsection{Performance with the non-trained Nine-Hole-Peg-Test (NHPT)}

The ANOVA for repeated measures indicated a differences between right and left hand performance 
Table 1

Principal component loadings (mean and 95\% CI) for repeated arm ability task measures during training

\begin{tabular}{lccccc}
\hline & Component 1 & Component 2 & Component 3 & Component 4 & Component 5 \\
\hline Variance explained & 31.9 & 31.5 & 27.8 & 27.3 & 21.4 \\
\hline Task & & & & & \\
$\quad$ Aiming & $\mathbf{8 8 . 8}(84.1,93.5)$ & $4.6(-1.0,10.2)$ & $2.2(-6.5,10.9)$ & $-23.5(-30.0,-17.1)$ & $3.0(-6.7,12.7)$ \\
Tapping & $-13.5(27.6,0.5)$ & $13.4(-3.5,30.3)$ & $\mathbf{6 1 . 5}(47.4,75.6)$ & $-22.8(-41.2,-4.4)$ & $3.3(-9.1,15.7)$ \\
Crossing circles & $2.6(-4.1,9.4)$ & $\mathbf{8 9 . 8}(85.1,94.5)$ & $9.8(1.6,18.0)$ & $-12.6(-22.5,-2.8)$ & $-4.7(-14.2,4.7)$ \\
Turning coins & $36.2(17.7,54.7)$ & $33.7(25.4,41.3)$ & $10.7(-4.1,25.4)$ & $-26.8(-37.7,-15.8)$ & $-42.9(-53.0,-32.8)$ \\
Labyrinth & $-28.9(-38.1,-19.7)$ & $-7.2(-17.3,2.9)$ & $0(-10.6,10.6)$ & $\mathbf{7 8 . 8}(69.1,88.4)$ & $-1.3(-15.9,13.3)$ \\
Nuts and bolts & $-6.2(-13.9,1.5)$ & $41.1(30.6,51.5)$ & $-\mathbf{6 0 . 6}(-72.5,-48.7)$ & $14.4(2.0,26.8)$ & $27.8(15.0,40.5)$ \\
Placing small obj. & $7.7(-10.8,26.2)$ & $2.1(-15.2,19.5)$ & $-30.2(-49.5,-10.9)$ & $31.9(18.1,45.8)$ & $-19.1(-36.3,-1.9)$ \\
Placing large obj. & $37.4(24.7,50.0)$ & $25.6(9.3,41.9)$ & $12.5(-3.7,28.7)$ & $17.9(7.7,28.2)$ & $54.5(40.9,68.0)$ \\
\hline
\end{tabular}

Table 1 depicts the variance explained by each factor and the total communality estimates (first row) as well as the distribution of loadings of the five principal components for variables of each of the 8 Arm Ability training tasks ( 4 block $\times 5$ days [except for block 1 on day 1] = 19 variables per task). Presented are mean values for the 19 variables for each task and the $95 \%$ confidence intervals. Mean values $>60$ are highlighted. The data shows a considerable to high loading of component 1 on aiming, of component 2 on crossing circles, of component 3 on tapping and 'bolts and nuts', and of component 4 on the labyrinth task.

(factor 'hand', $\mathrm{F}(1,6)=16.31 ; p=0.0068$ ) and an improved performance on this non-trained task across days (mean and s.d. of time needed for the NHPT: baseline-right hand $15.17 \mathrm{~s}$; baseline-left hand $16.28 \mathrm{~s}$; day 5-pre stim-right hand $13.26 \mathrm{~s}$; day 5-pre stim-left hand $14.77 \mathrm{~s}$ ) (factor 'days', $\mathrm{F}(4,24)=9.01 ; p(\mathrm{H}-$ $\mathrm{F})=0.0006 ; \mathrm{d}=1.23$ ). Improvements over days were not different for right or left hand; there were no within session improvements in performance.

\subsection{Effects of cTBS-600}

The results above indicate that improvement of performance occurred within each session from the 1st half of the session (blocks $1 \& 2$ for each task) to the 2nd half of the session (blocks $3 \& 4$ for each task). These change scores between the 1st and 2nd half of each training session (standardised with the baseline data for each task) were analysed with negative values indicating improvement rates and positive values indicating deterioration. The grand average of standardised change scores across all tasks and days 2 to 5 had been -0.021 (95\% CI: - .017--.025), indicating a within-session improvement of $2.1 \%$ on average.

When the grand average of change scores across all 8 training tasks was analysed, the repeated measures ANOVA revealed that the mean improvement rates from block $1 \& 2$ to block $3 \& 4$ within each session were influenced by the cTBS site $(\mathrm{F}(4,24)=9.16$; $p<0.0001 ; \mathrm{d}=1.24)$. The pre-specified analyses of the transformation matrix indicated that changes after cTBS over S1 significantly differed from the mean changes after cTBS over M1, PMC, and SMA
Table 2

Final performance with the Arm Ability training tasks after 5 days of training

\begin{tabular}{lc}
\hline Task & Mean 95\% CI \\
\hline Aiming & $0.83(0.69,0.96)$ \\
Tapping & $0.95(0.89,1.01)$ \\
Crossing circles & $0.65(0.53,0.77)$ \\
Turning coins & $0.78(0.70,0.85)$ \\
Labyrinth & $0.81(0.68,0.94)$ \\
Nuts and bolts & $0.77(0.56,0.99)$ \\
Placing small obj. & $0.69(0.60,0.79)$ \\
Placing large obj. & $0.81(0.76,0.86)$ \\
Grand average & $0.79(0.73,0.84)$ \\
\hline
\end{tabular}

Table 2 depicts the performance for each of the 8 Arm Ability training tasks and their grand average at the end of training (block 4 on day 5). Presented are mean values of standardised scores and the $95 \%$ confidence intervals. Standardisation was done with scores from each task at the beginning of training (1st block, day 1). Values $<1.0$ indicate improved performance.

$(\mathrm{F}(1,6)=6.53 ; p=0.0432 ; \mathrm{d}=1.04)$ (see also Fig. 5A). The contrast was.009 indicating a detrimental effect; i.e. a differential detrimental effect on changes within a session of the magnitude of $0.9 \%$ was attributable to cTBS over S1.

The effect of cTBS over different stimulation sites on change scores was also analysed for each of the Arm Ability tasks separately (see also Fig. 5B).

Tapping was markedly affected by cTBS showing on average a lack of within session improvement after cTBS; the cTBS effects on tapping varied across stimulation sites with the contrast for M1 being significant $(\mathrm{F}(1,6)=7.47 ; p=0.0341 ; \mathrm{d}=2.73)$. The magnitude of the contrast was.004 indicating a detrimental effect. 


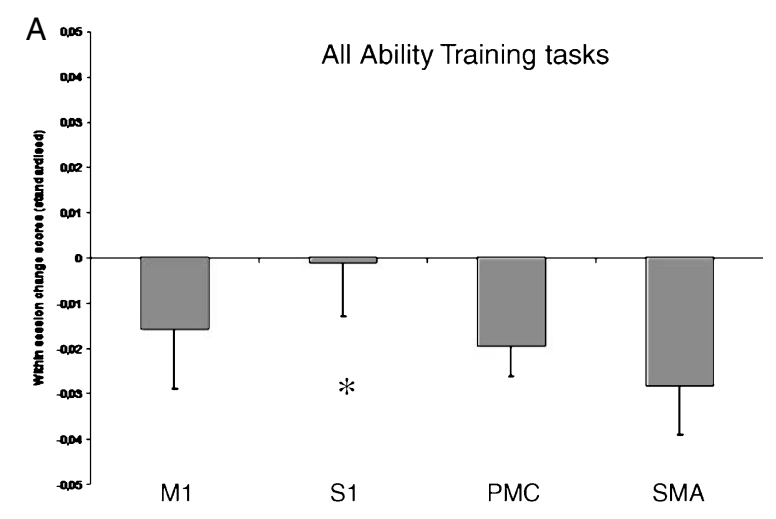

B
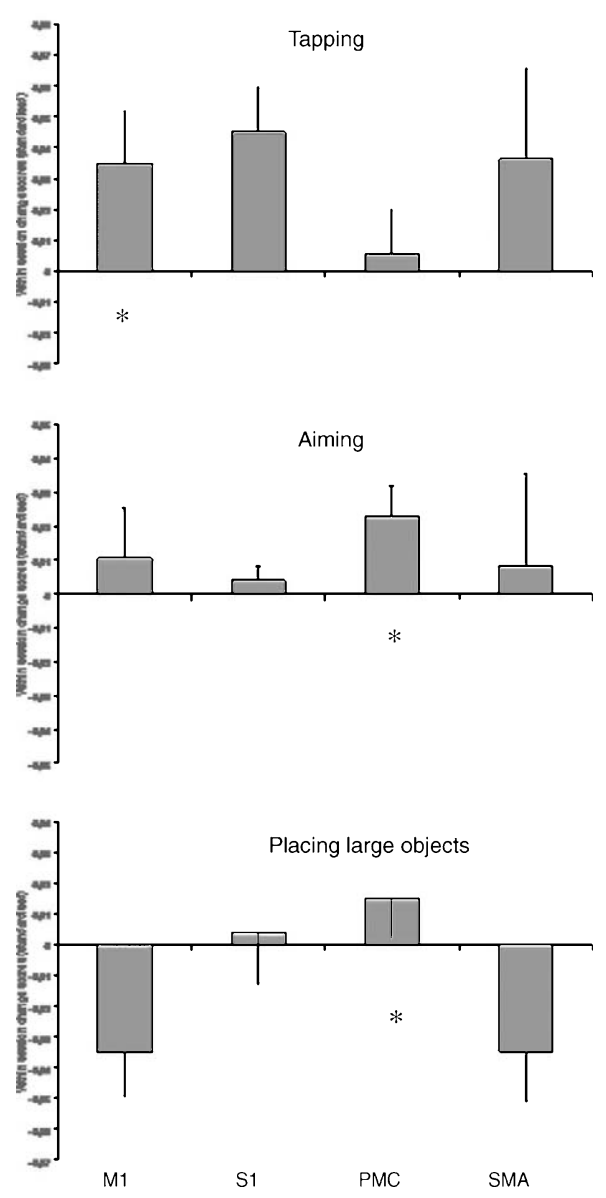

For aiming there was a differential effect of cTBS over PMC as compared to the mean changes after cTBS over M1, S1, and SMA $(\mathrm{F}(1,6)=8.82 ; p=0.0249$; $\mathrm{d}=2.97)$; the contrast was 0.004 indicating a detrimental effect.

Similarly, placing large objects was differentially affected by the site of stimulation with the contrast for stimulation over PMC $(\mathrm{F}(1,6)=8.83 ; p=0.0249$; $\mathrm{d}=1.21$ ) having significantly different effects compared to the mean of the other stimulation sites. The contrast was 0.011 indicating a detrimental effect. In addition, the data suggests that within session improvements with placing large objects were not impaired, but could have been enhanced with cTBS over M1 and SMA (compare Fig. 5B).

With crossing circles, turning coins, labyrinth tracking, bolts and nuts, and placing small objects no differential effect of any of the stimulation sites as compared to the mean of the other stimulation sites could be corroborated statistically.

There was no evidence that within session changes for the Nine-Hole-Peg-Test performed with either hand were differentially affected by the different stimulation sites.

MEP amplitudes and latencies of MEPs from either hemisphere were not differentially affected by cTBS over different stimulation sites.

\subsection{Side effects}

One subjects suffered from mild headaches for a couple of hours and a transient subjective impression

Fig. 5. Effects of cTBS. Fig. 5A presents grand average data across all eight Arm Ability tasks, Fig. 5B data for individual training tasks. Data is presented as mean and standard error of the mean (error bars) of changes scores from the 1st half of the training session before cTBS to the 2nd half of a training session after cTBS. Negative values indicate an (continued) improvement despite the cTBS, positive values indicate deterioration of performance after cTBS. Differential effects of cTBS over different brain sites (i.e. M1, S1, PMC, and SMA) had been analysed statistically; symbols indicate the level of significance for the contrast of a specific sites as compare to the three other sites: ${ }^{*} p<0.05$. Data for all tasks (grand average, Fig. 5A) indicated a more negative effects of cTBS over S1 as compared to the other stimulation sites. When data of individual tasks had been analysed task-specific effects of stimulation site became evident: Learning of finger tapping speed was most affected by cTBS, especially over M1, and ballistic arm navigation in extra-personal space (aiming and placing large objects) by cTBS over PMC; at the same time cTBS over M1 and SMA seemed rather to enhance performance with placing large objects. 
of having difficulties to focus with her eyes ('as if her eyes were wet') after her first cTBS session (SMA); she was discontinued from the study and replaced.

\section{Discussion}

\subsection{Motor learning}

In this experiment we sought for one to investigate the behavioural learning dynamics of the Arm Ability Training (AAT) in healthy subjects.

The behavioural data shows that AAT not only improves performance of patients with reduced fine motor skill after traumatic brain injury or stroke (Platz et al., 2001; Platz et al., 2009), but also improves performance of young healthy subjects who train their non-dominant arm. AAT improved motor performance within sessions and across five days of training (effect size 2.28). At the end of training standardised scores had improved from 1.0 at baseline to 0.79 on average at the end of the last session (95\% CI:0.73-0.84) indicating a $20 \%$ improvement over 5 days. This considerable training effect that built up over days indicates substantial motor learning in these healthy subjects.

Interestingly, performance improved even in the non-trained Nine-Hole-Peg-Test (NHPT) pointing to a transfer of skilfulness across tasks (effect size 1.23). This is an important observation since transfer to a non-trained task as here or retention over time have conventionally been considered as evidence of motor learning and not only improvement of skilfulness with a trained task (Schmidt, 1975; Shea and Wulf, 2005). If the AAT induced motor learning across different sensorimotor abilities it would be likely that other arm motor tasks like the NHPT that also afford these abilities would show improved performance after the AAT. Similarly robust effects of the AAT on non-trained motor tasks had been shown in clinical trials (Platz et al., 2001; Platz et al., 2009). Some effect of practice might, however, have occurred by repeated testing of the NHPT. The fact that NHPT performance did not only improve with the trained left arm, but also the non-trained right arm favours the notion that the enhanced task performance involved - to some extent - effector-independent processes.

The overall robust motor learning effect was mirrored by much smaller, yet detectable and significant improvements of performance that occurred within each training session (on average $2.1 \%$ ) that were used to analyse any immediate effects of cTBS on these learning dynamics within sessions.

\subsection{Motor abilities}

The study further assessed whether there is explicit behavioural evidence in favour of the assumption of different sensorimotor abilities that are trained.

The research question we addressed with the complete behavioural data set was whether motor performance and its changes over 5 days had a singular pattern across all AAT tasks or whether there had been different dynamics across different tasks? The latter would be an argument for distinct (independent) arm motor abilities that can be separately trained as might be suggested by our knowledge of the modular cerebral motor control (Jeannerod, 1997; Schieber, 1999). The former would suggest a more generalised structure of motor learning.

Both the PCA results with 5 strong uncorrelated components and the differential loading of the AAT tasks on these components as well as the analysis of variance of the variable training-induced changes across AAT tasks together with the differential final level of performance with the AAT tasks all pointed to independent components of motor performance and skill learning.

The data are consistent with the assumption that there are separate components of fine motor skill such as aiming ("aiming task"), steadiness ("crossing circles" task), visuomotor tracking ("labyrinth" task), and the ability of fast selective finger movement control ("tapping") versus precision grip control ("bolts and nuts" task) that respond differentially to training. Following this line of argument the other trained tasks seemed to involve a mixture of abilities since their scores were less clearly linked to one component, but rather to several (compare Table 1). The (simple) ability to make fast finger movements ("tapping"), an ability that presumably relies on M1 control mainly showed the least improvement at the end of training (Table 2), while all other tasks involving a higher degree of sensorimotor integration showed a larger improvement, which again varied across tasks. It is noteworthy, that the arm motor tasks that require a higher degree of sensorimotor integration had been more responsive to training than the more basic control ability to make fast selective finger movements.

In summary, the behavioural data support the notion that (1.) the AAT training improves independent 
sensorimotor abilities, and that (2.) substantial motor learning including transfer to a non-practiced task and the non-trained limb takes place. On a theoretical account this implies AAT training may have induced improved representation of different classes of motor performance, i.e. "generalised motor programs" (GMP), that at least to some extent involve effector-independent aspects.

\subsection{Effects of rTMS on motor learning}

Finally, the study investigated to what extent selected cortical areas are involved in producing within session task specific improvements. To address this question we used cTBS to interfere focally with neural processing in each of these brain regions during training and measure the behavioral consequences on each of the different motor abilities.

cTBS was applied during each training session on days 2 to 5 over either M1, S1, PMC or SMA (order was counterbalanced across subjects) and its effect on motor learning was analysed with intra-session effects which examined how cTBS changed performance from the 1 st to the 2 nd half of each training session. Thus, only small yet significant incremental improvement rates in the range of $2 \%$ on average served as a basis for comparison. There was no sham-stimulation session (sham coil or stimulation over non-motor area) since the research question was not to document any cTBS effect, but rather to investigate cTBS effects that were specific for a certain cortical area as compared to other motor-related brain regions. Accordingly, the four stimulation sites were compared against each other; the mean of all other stimulation sites served as control condition for each individual stimulation site. The data revealed significantly differential effects of cTBS between the 4 cortical areas. Overall, this approach revealed informative results with large effect sizes $(d=1.04-2.97)$.

When effects of cTBS were analysed across all tasks (grand average), stimulation over S1 had a detrimental effect that differed significantly from the three other stimulation sites, i.e. M1, PMC, and SMA (effect size 1.04; differential detrimental effect for cTBS over S1: $+0.9 \%$ ) (see Fig. 5A). Since all tasks involved some form or sensorimotor integration this was perhaps not surprising. For example, Vidoni et al. (2010) showed recently that rTMS of S1 impaired motor learning on a tracking task that involved precise integration of somatosensory feedback with the motor command.
Further analysis showed that cTBS over other cortical areas had effects on specific tasks (see Fig 5B). Performance during training of the tapping task was most impaired after cTBS, especially over M1 (effect size 2.73). Both aiming and placing large objects were specifically affected after cTBS over PMC (effect sizes 2.97 and 1.21, resp.). Further, with placing large objects cTBS over M1 and SMA rather seemed to enhance training effects (average improvement rates here were $3.5 \%$ as compared to the grand average of $2 \%$ ).

Thus, motor improvement with the two tasks that involved hand effector motion in extra-personal space (aiming and placing large objects) seemed to rely on intact PMC, an area that is thought to be involved in these processes (Fattori et al., 2010; Rickert et al., 2009). Similarly, speed of finger movements is well known to involve the primary motor cortex (Koeneke et al., 2006; Riecker et al., 2003); thus, interference with its function was expected to impair skill improvement with the "tapping" task.

Overall, the cTBS effects on individual tasks are suggestive of a pattern where cTBS over M1 and SMA deteriorated speed of isolated finger movements while at the same time enhancing improvement with a relatively coarse movement of the arm in extrapersonal space that conversely was affected by cTBS over PMC (compare Fig. 5B). This observation further supports the assumption of different arm abilities that rely on different cortical motor areas and show different dynamics during early motor learning (within a few days of practice).

It is worth noting that these differential effects of cTBS on motor behaviour could only be distinguished because the experiment used a combination of subthreshold focal cTBS to target the activity of restricted cortical areas together with an analysis that addressed specific sensorimotor demands (arm ability training tasks) and identified specific behavioural dynamics (within session improvements). Changes in dynamics that occurred during early motor learning before overlearnt automatic skill consolidation could be assumed to have taken place (compare Fig. 4) and in different sensorimotor control abilities were critical in detecting these differential cTBS effects in these young healthy subjects.

Performance of a standardised skilled motor task (like the Nine-Hole-Peg-Test) that was not trained was not (differentially) affected by rTMS over different brain areas. 


\subsection{Clinical considerations}

The AAT was designed as a specific, highly structured, and with regard to abilities a comprehensive training method for patients with mild arm paresis (Platz, 2004) and proved to be more effective than individually selected conventional therapy in a recent multicentre trial (Platz et al., 2009).

A major motivation for this study was to learn about the (physiological) effects and mechanisms of action of the AAT in healthy subjects. In patient populations, these would always be intermingled with both trainingindependent and training-modified, and thus complex brain recovery mechanisms.

Our study provides some insight about effects of the AAT in healthy subjects:

For one, it showed that the AAT both addresses and successfully induces substantial motor learning across distinct arm motor abilities with transfer to a non-trained task.

Secondly, a closer look at within training session dynamics and their experimental modification by neuronavigated focal cTBS suggested that the AAT differentially involves motor-related brain regions (M1, SMA, PMC, S1) in an early stage of motor learning.

Both its comprehensiveness and its ability to induce motor learning might contribute to its superior clinical efficacy compared to conventional, less systematically designed motor training in stroke rehabilitation (Platz et al., 2009).

Furthermore, the specific functional involvement of different cortical regions within the human motor systems for improving different motor abilities as revealed in the current study may be instrumental for developing new and more specific brain-system based TMS protocols to guide motor recovery after stroke.

\section{Conclusion}

This study on early motor learning indicated that the Arm Ability Training induces substantial motor learning in young healthy subjects by improving performance of different independent motor abilities, i.e. aiming, speed, steadiness, and visuomotor tracking. Motor-related cortical areas such as M1, SMA and PMC seem to be differentially involved in early motor learning across these arm motor abilities, e.g. M1 for speed and PMC for the ballistic arm navigation in extra-personal space. The integrity of function of the primary somatosensory cortex (S1) seemed to be relevant for motor learning across abilities.

\section{Acknowledgments}

The research leading to these results has received funding from the European Union (ImpactG Project, FP7-REGPOT-2008-1, Grant agreement no.: 229750, awarded to T.P.), the European Research Council under the European Union's Seventh Framework Programme (FP7/2007-2013) / ERC Grant agreement $n^{\circ}$ [263472], awarded to A.T.S.), and by the German Research Association (DFG; LO 795/7, awarded to T.P.). The sponsors had no role in study design; in the collection, analysis, and interpretation of data; in the writing of the report; or in the decision to submit the paper for publication. The help of both our volunteers and the certified TMS assessors from the Department of Cognitive Neuroscience, Faculty of Psychology and Neuroscience, Maastricht University, namely Nina Bien, Christianne Jacobs, Tom de Graaf, Joel Reithler, Vincent van de Ven, Teresa Schuhmann, with TMS assessment and cTBS is gratefully acknowledged.

\section{References}

Ashe, J., Lungu, O.V., Basford, A.T. \& Lu, X. (2006). Cortical control of motor sequences. Curr Opin Neurobiol, 16, 213-221.

Baraduc, P., Lang, N., Rothwell, J.C. \& Wolpert, D.M. (2004). Consolidation of dynamic motor learning is not disrupted by rTMS of primary motor cortex. Current Biology, 14, 252-256.

Bezzola, L., Mérillat, S., Gaser, C. \& Jäncke, L. (2011). Traininginduced neural plasticity in golf novices. J Neurosci, 31, 1244412448.

Brashers-Krug, T., Shadmehr, R. \& Bizzi, E. (1996). Consolidation in human motor memory. Nature, 382, 252-255.

Cohen, J. (1988). Statistical power analysis for behavioural sciences (2nd edition). Erlbaum, Hillsdale. NJ.

Eickhof, C. (2001). Grundlagen der Therapie bei erworbenen Lähmungen, München, Pflaum Verlag, 160-213.

Fattori, P., Raos, V., Breveglieri, R., Bosco, A., Marzocchi, N. \& Galletti, C. (2010). The dorsomedial pathway is not just for reaching: Grasping neurons in the medial parieto-occipital cortex of the macaque monkey. Journal of Neuroscience, 30 , 342-349.

Grafton, S., Hazeltine, E. \& Ivry, R. (2002). Motor sequence learning with the nondominant left hand. Exp Brain Res, 146, 369-378.

Hankey, G.J., Jamrozik, K., Broadhurst, R.J., Forbes, S. \& Anderson, C.S. (2002). Long-term disability after first-ever stroke and related prognostic factors in the Perth community stroke study, 1989-1900. Stroke, 33, 1034-1040. 
Huang, Y.Z., Edwards, M.J., Rounis, E., Bhatia, K.P. \& Rothwell, J.C. (2005). Theta burst stimulation of the human motor cortex. Neuron, 45, 201-206.

Jäncke, L. (2009). The plastic human brain. Restor Neurol Neurosci, 27, 521-538.

Jeannerod, M. (1997). The cognitive neuroscience of action, Oxford, Blackwell.

Karni, A., Meyer, G., Jezzard, P., Adams, M.M., Turner, R. \& Ungerleider, L.G. (1995). Functional MRI evidence for adult motor cortex plasticity during motor skill learning. Nature, 377, 155158.

Keetch, K.M., Lee, T.D. \& Schmidt, R.A. (2008). Especial skills: Specificity embedded within generality. Journal of Sport and Exercise Psychology, 30, 723-736.

Kobayashi, M., Théoret, H. \& Pascual-Leone, A. (2009). Suppression of ipsilateral motor cortex facilitates motor skill learning. European Journal of Neuroscience, 29, 833-836.

Koeneke, S., Lutz, K., Herwig, U., Ziemann, U. \& Jäncke, L. (2006). Extensive training of elementary finger tapping movements changes the pattern of motor cortex excitability. Experimental Brain Research, 174, 199-209.

Maas, E., Robin, D.A., Austermann Hula, S.N., Freedman, S.E., Wulf, G., Ballard, K.J. \& Schmidt, R.A. (2008). Principles of motor learning in treatment of motor speech disorders. Am J Speech Lang Pathol, 17, 277-298.

Nakayama, H., Jorgensen, H.S., Raaschou, H.O. \& Olsen, T.S. (1994). Recovery of upper extremity function in stroke patients: The Copenhagen Study. Archives of Physical Medicine and Rehabilitation, 75, 394-398.

Park, J.W., Kim, Y.H., Jang, S.H., Chang, W.H., Park, C.H. \& Kim, S.T. (2010). Dynamic changes in the cortico-subcortical network during early motor learning. NeuroRehabilitation, 26, 95-103.

Pascual-Leone, A., Grafman, J. \& Hallett, M. (1994). Modulation of cortical motor output maps during development of implicit and explicit knowledge. Science, 263, 1287-1289.

Platz, T. (2004). Impairment-oriented Training (IOT) - scientific concept and evidence-based treatment strategies. Restorative Neurology and Neuroscience, 22, 301-315.
Platz, T., Winter, T., Müller, N., Pinkowski, C., Eickhof, C. \& Mauritz, K.-H. (2001). Arm Ability Training for Stroke and Traumatic Brain Injury Patients with mild arm paresis. A Single-Blind, Randomised, Controlled Trial. Archives of Physical Medicine and Rehabilitation, 82, 961-968.

Platz, T., van Kaick, S., Mehrholz, J., Leidner, O., Eickhof, C. \& Pohl, M. (2009). Best conventional therapy versus modular Impairment-oriented training (IOT) for arm paresis after stroke: A single blind, multi-centre randomized controlled trial. Neurorehabilitation and Neural Repair, 23, 706-716.

Rickert, J., Riehle, A., Aertsen, A., Rotter, S. \& Nawrot, M.P. (2009). Dynamic encoding of movement direction in motor cortical neurons. Journal of Neuroscience, 29, 13870-13882.

Riecker, A., Wildgruber, D., Mathiak, K., Grodd, W. \& Ackermann, H. (2003). Parametric analysis of rate-dependent hemodynamic response functions of cortical and subcortical brain structures during auditorily cued finger tapping: A fMRI study. Neuroimage, 18, 731-739.

Rossi, S., Hallett, M., Rossini, P.M., Pascual-Leone, A. \& The Safety of TMS Consensus Group. (2009). Safety, ethical considerations, and application guidelines for the use of transcranial magnetic stimulation in clinical practice and research. Clinical Neurophysiology, 120, 2008-2039.

Schieber, M.H. (1999). Voluntary descending control. In: Zigmond, M.J., Bloom, F.E., Landis, S.C., Roberts, J.L., and Squire, L.R. Eds. Fundamental Neuroscience, San Academic Press, Diego, 931-949.

Schmidt, R.A. (1975). A schema theory of discrete motor skill learning. Psychological Review, 82, 225-260.

Shea, C.H. \& Wulf, G. (2005). Schema theory: A critical appraisal and reevaluation. $J$ Mot Behav, 37, 85-101.

Vidoni, E.D., Acerra, N.E., Dao, E., Meehan, S.K. \& Boyd, L.A. (2010). Role of the primary somatosensory cortex in motor learning: An rTMS study. Neurobiology of Learning and Memory, 93, 532-539. 